\title{
Power Allocation and Hybrid Relaying Strategies for F/TDMA Ad Hoc Networks
}

\author{
Semih Serbetli and Aylin Yener \\ Wireless Communications and Networking Laboratory \\ Electrical Engineering Department \\ The Pennsylvania State University \\ University Park, PA 16802 \\ serbetli@psu.edu \\ yener@ee.psu.edu
}

\begin{abstract}
In this paper, we study the power allocation problem at the relay nodes for two-hop F/TDMA networks with multiple sources and destinations. Each relay node is assumed to be capable of assisting multiple source nodes and of selecting one of regenerative decode-and-forward (RDF), nonregenerative decode-and-forward (NDF), amplify-and-forward (AF) and compress-and-forward (CF) relaying strategies to assist each source node. Considering the sum capacity as the performance metric, we solve the problem of optimally allocating the total power of each relay node between the transmissions it is assisting. We first obtain the optimum power allocation policies for the relay nodes when the relaying strategies of all source nodes are given. Next, to obtain higher sum capacities, we investigate the optimum power allocation problem jointly with relaying strategy selection. We observe that optimum power allocation with the appropriate hybrid relaying strategies provides higher sum capacity than pure RDF, NDF, AF or CF relaying strategies.
\end{abstract}

\section{INTRODUCTION}

Wireless ad hoc networking is an emerging technology for next generation wireless systems due to its ability of providing communication among the nodes without the need for infrastructure [1], [2]. Yet, serious design challenges exist for wireless ad hoc networks including effectively combating the impairments of the wireless channel. Relay assisted communications, where intermediate nodes help forward traffic from source nodes to their destinations, exploits spatial diversity without needing to deploy physical antenna arrays [3]-[12]. Relay assistance also mitigates the effects of path loss, provides the source nodes with extended battery life [7]-[10], and helps extending coverage [10], [13], [14]. An intense research effort is underway to better understand the performance of relay assisted schemes.

Results related to the capacity of the full duplex relay channel go back to [3], [4]. Since it is difficult to have the nodes transmit and receive simultaneously in the same frequency, much of the recent research effort is towards investigating orthogonal relay transmission schemes where the source and relay nodes transmit in orthogonal channels [5], [7]-[10], [12]. Recently, reference [6] showed that the uplink capacity of twouser systems can be increased by using cooperation, where each user also acts as a relay for the other.

In wireless networks, transmission power of the nodes is limited. Hence, power efficiency is a critical concern when designing relay transmission strategies. It has been shown that significant performance improvement is achieved by the optimum power allocation for various relay assisted networks with single source destination pair [7], [9]-[11].

Relay assisted transmission is expected to improve the performance of multiuser systems as well [15], [16]. Such networks, henceforth referred to as multiuser relay networks are ones where each relay node would serve multiple users (source nodes), and the total transmission power budget for each relay node would be limited. When this is the case, each source node's transmission should be relayed with a fraction of the power from its corresponding relay node. In such a scenario, the total relay power should be allocated between the transmissions of information from the sources that relay over this node, in order to obtain the best performance. The optimum power allocation for the relay nodes is studied for single source-destination pair in [7], [9]. In [17], the power allocation problem for the relay nodes in F/TDMA ad hoc networks is investigated for the case when each relay node assists the sources via the same relaying strategy. In this paper, we extend the work in [17] to multiuser relay networks with nodes capable of employing hybrid relaying strategies, i.e., we allow each relay node to adopt a distinct relaying strategy for each source it assists. It is assumed that the relay nodes assist the source nodes employing one of decode-and-forward [5], [7], amplify-and-forward [5], compress-and-forward [16] relaying strategies for each source node. We first address the power allocation problem for given relaying strategies of all source nodes. Motivated by obtaining higher sum capacities, we next investigate the optimum power allocation problem with relaying strategy selection where the relay node can also choose the relaying strategies for the source nodes it will be assisting. Our objective in this work is to find the optimum power allocation as well as the relaying strategies for all source nodes assisted by the relay nodes considering the sum capacity as the performance metric. We observe that the hybrid relaying strategies along with power allocation results in higher sum capacity then pure RDF, NDF, AF or CF.

\section{SYSTEM MODEL}

We consider a relay assisted F/TDMA ad hoc network with $K$ source nodes and $L$ relay nodes. We assume that 


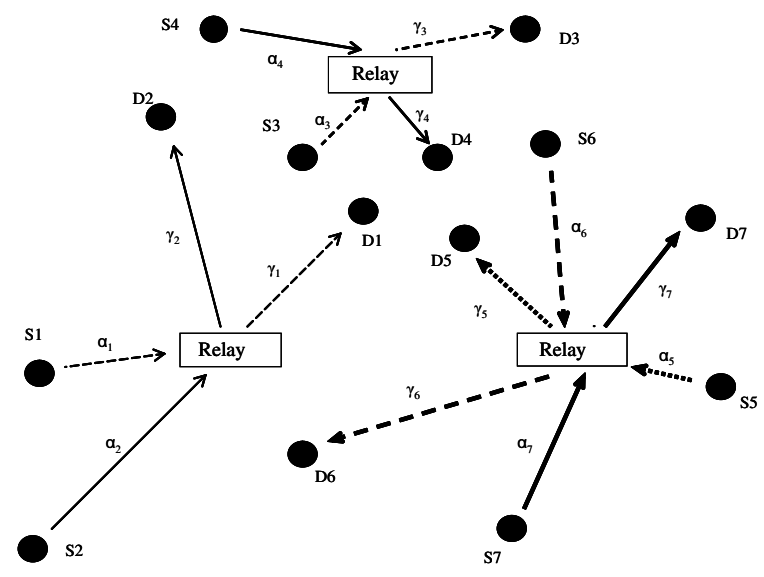

Fig. 1. System Model

each source node intends to transmit its signal to a different destination and has a pre-assigned relay node that will assist its transmission. The data transmission of each source node occurs in two pre-assigned channels that can be either time slots or different frequencies. The source node broadcasts its signal in the first channel, and the preassigned relay node transmits this source node's information in the second channel. All channels of all source nodes and relay nodes are distinct and nonoverlapping. The signal received by the destination in the $i$ th source node's first channel is

$$
y_{d i 1}=\sqrt{P_{s i}} \beta_{i} x_{s i}+n_{d i 1}
$$

where $x_{s i}$ is the symbol transmitted by source node $i, P_{s i}$ is the transmit power of source node $i$ and $\beta_{i}$ denotes the normalized channel gain from source node $i$ to the destination with $n_{d i 1}$ as the zero mean AWGN with unit variance. Similarly, the received signal at the relay node $k$ to which source node $i$ is assigned, is

$$
y_{r i}=\sqrt{P_{s i}} \alpha_{i} x_{s i}+n_{r i}
$$

where $\alpha_{i}$ is the normalized channel gain from source node $i$ to the assigned relay node $k$, and $n_{r i}$ is the zero mean AWGN with unit variance. In the second channel of the $i$ th source node, the $k$ th relay node transmits $x_{r i}$, and the corresponding received signal at the destination is

$$
y_{d i 2}=\sqrt{P_{r i}} \gamma_{i} x_{r i}+n_{d i 2}
$$

where $x_{r i}, P_{r i}$ and $\gamma_{i}$ denote the signal transmitted for source node $i$ from the $k$ th relay node, the transmit power of the $k$ th relay node dedicated to source node $i$ and the normalized channel gain from the $k$ th relay node to the destination of the $i$ th source node with a zero mean and unit variance AWGN $n_{d i 2}$, respectively. Note that the relay node should transmit after the source due to causality constraints, and this constraint results in loss of one time slot when the channels represent different frequencies. We assume that each relay node has a total power constraint $\sum_{i \in A_{k}} P_{r i} \leq P_{R k, t o t a l}$ where $A_{k}$ denotes the set of source nodes that relay their information through node $k$. We consider four different relaying strategies at the relay nodes.
- Regenerative Decode-and-Forward (RDF): When the transmission from the source node is received reliably at the relay node, the relay node decodes the signal, reencodes it with the same codebook used in the original source node's transmission and transmits the signal in the second channel of the source node [5], [9], [10].

- Nonregenerative Decode-and-Forward (NDF): Similar to RDF, the relay decodes the signal, but re-encodes it with a codebook different than the original source node and transmits it in the second channel of the source node [7].

- Amplify-and-Forward (AF): The signal received at the relay node is amplified and forwarded in the second channel of the source node [5], [10].

- Compress-and-Forward (CF): In this model, the relay node compresses and forwards the source's signal in the second channel of the source node [16], [18].

For the clarity of exposition, we denote the sets of source nodes assisted by the $k$ th relay via $\mathrm{RDF}, \mathrm{NDF}, \mathrm{AF}$ and $\mathrm{CF}$ relaying as $A_{k, R D F}, A_{k, N D F}, A_{k, A F}$ and $A_{k, C F}$, respectively, and $A_{k, R D F} \cup A_{k, N D F} \cup A_{k, A F} \cup A_{k, C F}=A_{k}$.

\section{Optimal Power Allocation}

In this work, we aim to optimally distribute the power of each relay node between the source nodes' transmissions to be relayed by that node. Our goal is to maximize the sum capacity of the system. Clearly, the individual capacities of the source nodes are a function of the relaying strategies used. For given relaying strategies of all source nodes, i.e., $A_{k, R D F}, A_{k, N D F}$, $A_{k, A F}$ and $A_{k, C F}$, the optimum power allocation problem at the relay nodes is posed as

$$
\begin{aligned}
& \underset{\left\{P_{r i}\right\}_{i=1, \cdots, K}}{\max } C_{\text {sum }}=\sum_{k=1}^{L} \sum_{i \in A_{k}} C_{i, *} \\
& \text { s.t. } \sum_{i \in A_{k}} P_{r i} \leq P_{R k, \text { total }} ; \quad P_{r i} \geq 0 \quad \forall i, k
\end{aligned}
$$

where $C_{i, *}$ is the individual capacity of source node $i$ and * can be replaced with RDF, NDF, AF or CF according to the relaying strategy chosen, i.e., if $i \in A_{k, R D F}, C_{i, *}=$ $C_{i, R D F}$. We first address the optimal power allocation problem for given $A_{k, R D F}, A_{k, N D F}, A_{k, A F}$ and $A_{k, C F}$. Next, to obtain higher sum capacities, we remove this assumption in Section IV, and investigate the optimum power allocation problem with relaying strategy selection where the relay node chooses the best relaying strategy for each source node. Since the power allocation at each relay node does not affect the individual capacities of the source nodes that are served by other relay nodes, we focus on the sum capacity optimization problem at each relay node.

For both RDF and NDF, the designated relay node must reliably decode the signal. Thus, the individual capacity of a relay assisted source node cannot exceed the capacity of the source node to relay link. This constraint leads to several important results in terms of optimum power allocation. When the direct link, $\beta_{i}^{2}$, is better than the source node to relay 
link, $\alpha_{i}^{2}$ for source node $i$, the capacity of the direct link is higher than the capacity of the source to relay link. In this case, the direct transmission provides higher capacity than the relay assisted transmission for source node $i$. Since by employing direct transmission for source node $i$, the individual capacity of source node $i$ is maximized, and the relay has the potential to improve the sum capacity by investing its power in assisting the remaining source nodes, the relay power allocated to source node $i$ should be

$$
P_{r i}=0 \quad \text { if } \quad \alpha_{i}^{2} \leq \beta_{i}^{2}, \quad \forall i \in A_{k, R D F} \cup A_{k, N D F}
$$

In addition, observe that the maximum individual capacity of $i$ th source node is upper bounded by

$$
C_{i, R D F} \leq C_{i, N D F} \leq C_{\text {upperDF }, i}=\frac{1}{2 K} \log \left(1+P_{s i} \alpha_{i}^{2}\right)
$$

due to the decodability constraint at the relay node. Thus, allocating more power of the relay node for the transmission of a source node beyond a threshold will not increase the individual capacity of the source node. These constraints should be taken into account for the power allocation problem in RDF and NDF relaying strategies.

In the case of RDF relay transmission, the individual capacity of $i$ th source node is

$$
C_{i, R D F}=\min \left(\frac{1}{2 K} \log \left(1+P_{s i} \beta_{i}^{2}+P_{r i} \gamma_{i}^{2}\right), C_{\text {upperDF }, i}\right)
$$

Similarly, for the case of NDF relay transmission where the relay node uses a different codebook than the source node, i.e., $x_{r i} \neq x_{s i}$, we have

$C_{i, N D F}=\min \left(\frac{1}{2 K} \log \left(\left(1+P_{s i} \beta_{i}^{2}\right)\left(1+P_{r i} \gamma_{i}^{2}\right)\right), C_{\text {upperDF }, i}\right)$

When AF relay transmission is used, the individual capacity of $i$ th source node is

$$
C_{i, A F}=\frac{1}{2 K} \log \left(1+P_{s i} \beta_{i}^{2}+\frac{P_{s i} \alpha_{i}^{2} P_{r i} \gamma_{i}^{2}}{P_{s i} \alpha_{i}^{2}+P_{r i} \gamma_{i}^{2}+1}\right)
$$

In the case of CF relaying, when Gaussian codebooks are used, and the relay node compresses using Wyner-Ziv lossy source coding [19], the individual capacity of $i$ th source node can be expressed as [18]

$$
C_{i, C F}=\frac{1}{2 K} \log \left(1+P_{s i} \beta_{i}^{2}+\frac{P_{s i} \alpha_{i}^{2}}{1+\sigma_{W i}^{2}}\right)
$$

with

$$
\sigma_{W i}^{2}=\frac{P_{s i}\left(\alpha_{i}^{2}+\beta_{i}^{2}\right)+1}{P_{r i} \gamma_{i}^{2}\left(P_{s i} \beta_{i}^{2}+1\right)}
$$

For both $\mathrm{AF}$ and $\mathrm{CF}$ relaying, the individual capacities are upper bounded by

$$
C_{i, A F} \leq C_{i, C F} \leq \frac{1}{2 K} \log \left(1+P_{s i} \beta_{i}^{2}+P_{s i} \alpha_{i}^{2}\right)
$$

We are now ready to state our results for the optimal power allocation when relaying strategies of the source nodes are given.

Theorem 1: For the relaying strategy selection of the source nodes, $A_{k, R D F}, A_{k, N D F}, A_{k, A F}$ and $A_{k, C F}$, for the $k$ th relay node, the optimal relay power allocated to the source node $i$ is

$$
P_{r i}= \begin{cases}\min \left(\left(\frac{1}{\mu_{k}}-\frac{1+P_{s i} \beta_{i}^{2}}{\gamma_{i}^{2}}\right)^{+},\left(\frac{P_{s i}\left(\alpha_{i}^{2}-\beta_{i}^{2}\right)}{\gamma_{i}^{2}}\right)^{+}\right), & \text {if } i \in A_{k, R D F} ; \\ \min \left(\left(\frac{1}{\mu_{k}}-\frac{1}{\gamma_{i}^{2}}\right)^{+},\left(\frac{P_{s i}\left(\alpha_{i}^{2}-\beta_{i}^{2}\right)}{\gamma_{i}^{2}\left(1+P_{s i} \beta_{i}^{2}\right)}\right)^{+}\right), & \text {if } i \in A_{k, N D F} ; \\ \left(\frac{-\left(\frac{a_{i}}{b_{i}}+2\right)+\sqrt{\left(\frac{a_{i}}{b_{i}}\right)^{2}+\frac{4 a_{i}}{\mu_{k}}\left(1+\frac{a_{i}}{b_{i}}\right)}}{2\left(a_{i}+b_{i}\right)}\right)^{+}, & \text {if } i \in A_{k, A F} ; \\ \left(\frac{-\left(\frac{X_{i}}{Y_{i}}+2\right)+\sqrt{\left(\frac{X_{i}}{Y_{i}}\right)^{2}+\frac{4 X_{i}}{\mu_{k}}\left(1+\frac{X_{i}}{Y_{i}}\right)}}{2\left(X_{i}+Y_{i}\right)}\right)^{+}, & \text {if } i \in A_{k, C F} .\end{cases}
$$

with

$$
\begin{gathered}
a_{i}=\frac{P_{s i} \alpha_{i}^{2} /\left(P_{s i} \alpha_{i}^{2}+1\right)}{\left(1+\beta_{i}^{2} P_{s i}\right) / \gamma_{i}^{2}} ; \quad b_{i}=\frac{\gamma_{i}^{2}}{P_{s i} \alpha_{i}^{2}+1} \\
X_{i}=\frac{P_{s i} \alpha_{i}^{2} \gamma_{i}^{2}}{\left(P_{s i} \alpha_{i}^{2}+P_{s i} \beta_{i}^{2}+1\right)} ; \quad Y_{i}=\frac{\gamma_{i}^{2}\left(P_{s i} \beta_{i}^{2}+1\right)}{\left(P_{s i} \alpha_{i}^{2}+P_{s i} \beta_{i}^{2}+1\right)}
\end{gathered}
$$

where $\mu_{k}$ is the Lagrange multiplier associated with the total transmit power constraint of the relay node $k$ and (.) $)^{+}=$ $\max (., 0)$.

Proof: For given relaying strategies for the source nodes, the power allocation problem is a convex optimization problem since the capacity expressions for all relaying strategies are concave functions of relay powers, $\left\{P_{r i}\right\}$ and the set of relay powers is a convex set. Note that the decodability constraints of RDF and NDF relay yield the upper bounds for the relay power dedicated to the source node $i$ as

$$
\begin{gathered}
P_{r i} \leq \frac{P_{s i}\left(\alpha_{i}^{2}-\beta_{i}^{2}\right)}{\gamma_{i}^{2}}, \forall i \in A_{k, R D F} \\
P_{r i} \leq \frac{P_{s i}\left(\alpha_{i}^{2}-\beta_{i}^{2}\right)}{\gamma_{i}^{2}\left(1+P_{s i} \beta_{i}^{2}\right)} \forall i \in A_{k, N D F}
\end{gathered}
$$

Thus, the Lagrangian is

$$
\begin{aligned}
\mathrm{E}\left(\left\{P_{r i}\right\}, \mu_{k},\left\{\rho_{i}\right\},\left\{\zeta_{i}\right\}\right) & =\sum_{i \in A_{k, R D F}} C_{i, R D F}+\sum_{i \in A_{k, N D F}} C_{i, N D F} \\
+\sum_{i \in A_{k, A F}} C_{i, A F}+ & \sum_{i \in A_{k, C F}} C_{i, C F}+\hat{\mu}_{k}\left(P_{R k, t o t a l}-\sum_{i \in A_{k}} P_{r i}\right) \\
& +\sum_{i \in A_{k, R D F}} \rho_{i, R D F}\left(\frac{P_{s i}\left(\alpha_{i}^{2}-\beta_{i}^{2}\right)}{\gamma_{i}^{2}}-P_{r i}\right) \\
& +\sum_{i \in A_{k, N D F}} \zeta_{i, N D F}\left(\frac{P_{s i}\left(\alpha_{i}^{2}-\beta_{i}^{2}\right)}{\gamma_{i}^{2}\left(1+P_{s i} \beta_{i}^{2}\right)}-P_{r i}\right)
\end{aligned}
$$

where $\hat{\mu}_{k}, \rho_{i, R D F}$ and $\zeta_{i, N D F}$ are the Lagrange multipliers associated with the power constraint of the relay node $k$, the upper bound for the relay power used for source node $i$ in $\mathrm{RDF}$ relaying and the upper bound for the relay power used for source node $i$ in NDF relaying, respectively. Simply taking the derivative with respect to $P_{r i}$ and equating it to zero, we arrive at the optimum relay power for source node $i$ in (14) where $\mu_{k}=2 K \hat{\mu}_{k}$.

The optimum power allocation for hybrid relay nodes can be 
used for any relaying strategy of the source nodes. In the special case where all source nodes are assisted via the same relaying strategy, i.e., pure $\mathrm{RDF}, \mathrm{NDF}, \mathrm{AF}$ or $\mathrm{CF}$ relaying, the optimum power allocation of each case becomes a modified water-filling solution, and it is the identical to the optimum power allocation studied in [17].

\section{Relaying Strategy Selection}

So far we investigated the power allocation problem for given relaying strategies of the source nodes. Each relaying strategy has its own advantages and disadvantages, and one may perform better than the other in different scenarios. Thus, in principle, higher sum rates can be obtained if the appropriate relaying strategy is chosen for each source node. In such a scenario, each source node will be relayed with the relaying strategy that maximizes its individual capacity. This results in the individual capacity of source node $i$, $C_{i}=\max \left(C_{i, R D F}, C_{i, N D F}, C_{i, A F}, C_{i, C F}\right)$. Formally, the power allocation problem with relaying strategy selection is

$$
\begin{array}{r}
\max _{\left\{P_{r i}\right\}_{i=1}^{K}} C_{\text {sum }}= \\
\text { s.t. } \sum_{i=1}^{K} \max \left(C_{i, R D F}, C_{i, N D F} \leq C_{\text {ri, }} \leq P_{R k, t o t a l} ; P_{r i} \geq 0 \quad \forall i, k\right.
\end{array}
$$

Similar to Section III, we focus on the power allocation problem at each relay node. It is straightforward to see that each source node should choose either NDF or CF relaying strategy to maximize its individual capacity, i.e., $C_{i}=$ $\max \left(C_{i, N D F}, C_{i, C F}\right)$. The relaying strategy selection for each source node depends on $P_{r i}$ allocated for the transmission of the source node $i$ 's signal. Thus, the power allocation policy at the relay nodes dictates the relaying strategy to be used for each source node, and the total power of the relay node should be distributed appropriately considering these two relaying strategies. Unlike the case where the relaying strategies are fixed, the jointly optimum power allocation and relaying strategy selection is not a convex optimization problem. Using Theorem 1, the jointly optimum power allocation and relaying strategy selection can be found comparing the performance of $2^{K}$ possible relaying strategy selection scenarios each of which would have a corresponding power allocation policy. However, the computational complexity of such an approach is too high. Thus, we seek a low complexity near-optimum algorithm. To that end, we first investigate the conditions under which one of NDF or CF would be preferred over the other.

When the direct link of a source node is better than the source to relay link, $\beta_{i}^{2} \geq \alpha_{i}^{2}$, NDF relaying cannot improve the individual capacity of the source node resulting $C_{i}=$ $\max \left(C_{i, N D F}, C_{i, C F}\right)=C_{i, C F}$. Thus, the relay node should operate in CF mode for the source nodes with $\beta_{i}^{2} \geq \alpha_{i}^{2}$ and the set of such source nodes will be denoted as $A_{k, C F-\text { strict }}$ and the rest as $A_{k, C F-s t r i c t}^{\prime}$ in the sequel. The relaying strategies that the relay node should employ for the source nodes $\beta_{i}^{2}<$ $\alpha_{i}^{2}$ are dependent on $P_{r i}$. $C_{i}$ vs $P_{r i}$ performance of a source node with $\beta_{i}^{2}<\alpha_{i}^{2}$ is presented in Figure 2. For low $P_{r i}$

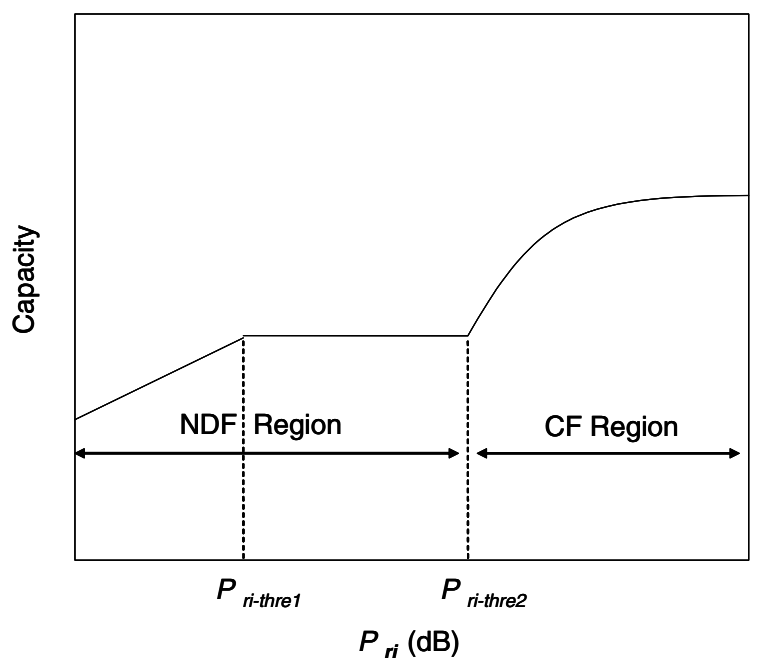

Fig. 2. Relay Power vs Capacity for Hybrid relaying

values NDF performs better than CF. At $P_{r i}=P_{r i-t h r e 1}=$ $\frac{P_{s i}\left(\alpha_{i}^{2}-\beta_{i}^{2}\right)}{\gamma_{i}^{2}\left(1+P_{s i} \beta_{i}^{2}\right)}$ NDF relaying achieves its maximum capacity, and increasing $P_{r i}$ further without changing the relaying strategy for source node $i$ does not increase the individual capacity of source node $i$. Up to $P_{\text {ri-thre } 2}=\frac{\left(P_{s i}\left(\alpha_{i}^{2}+\beta_{i}^{2}\right)+1\right)\left(\alpha_{i}^{2}-\beta_{i}^{2}\right)}{\gamma_{i}^{2} \beta_{i}^{2}\left(P_{s i} \beta_{i}^{2}+1\right)}$ NDF still outperforms CF. For $P_{r i}>P_{r i-t h r e 2}$, CF performs better than NDF for source node $i$. Thus, for low relay power scenarios, relaying all of the source nodes in $A_{k, C F-\text { strict }}^{\prime}$ with NDF and the rest with CF is a good relaying strategy, since it is likely that each source node will be allocated a relay power less than $P_{r i-t h r e 2}$. Similarly, for high relay power, one may choose to relay all the source nodes with CF since it is likely that each source node will be allocated a relay power higher than $P_{r i-t h r e 2}$. The global optimum power allocation with relaying strategy selection can be found via comparing the sum capacities of $2^{K-\left|A_{k, C F-\text { strict }}\right|}$ possible relaying strategy selection scenarios with their optimum power allocation.

Partitioning the source nodes into sets $A_{k, C F-s t r i c t}$ and $A_{k, C F-s t r i c t}^{\prime}$ for CF and NDF relaying and finding the optimum power allocation for such a relaying strategy selection can be a strong candidate for the jointly optimum power allocation and relaying strategy selection: If the optimum power allocation for such a partition results in all the NDF decodability constraints of the source nodes in $A_{k, C F-s t r i c t}^{\prime}$ being non-active, then the global optimum power allocation with relaying strategy selection is found. This is due to the fact that this solution is also the solution of the optimization problem when we relax the NDF decodability constraints of the original power allocation problem in (19) which provides an upper bound for the original problem. Such an approach is especially useful for low relay power scenarios where the probability of $A_{k, C F-s t r i c t}^{\prime}$ being the optimum $A_{k, N D F}$ is very high. If the NDF decodability constraints become active for some of the source nodes with such a relaying strategy, partitioning the source nodes into $A_{k, C F-\text { strict }}$ and $A_{k, C F-s t r i c t}^{\prime}$ sets for CF and NDF relaying, respectively, may 
TABLE I

Near Optimum Relaying Strategy SElection

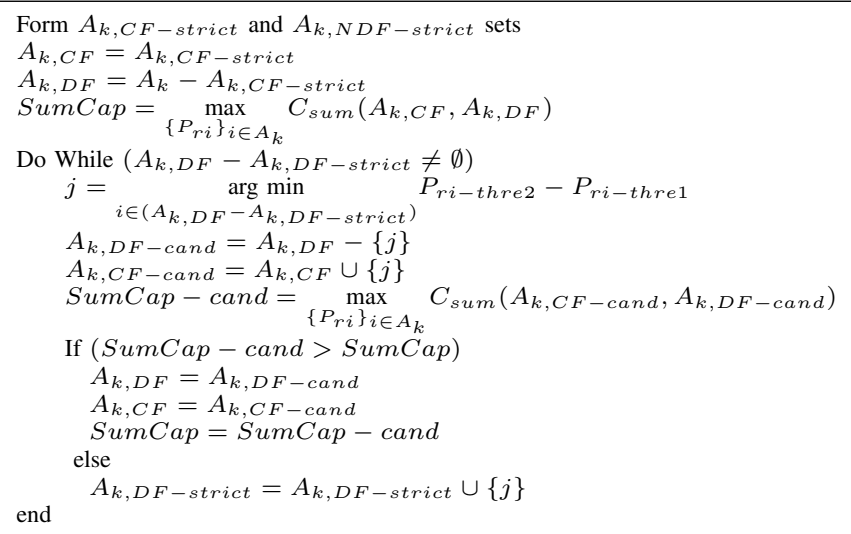

not be the optimum relaying strategy. However, investigating the optimal power allocation policy for such a partition provides insight towards the optimum relaying strategy. It is important to note that more relay power should be dedicated to a source node's transmission if it is relayed with CF relaying strategy. Thus, if a source node switches from NDF to CF, then less relay power will be allocated to the remaining source nodes. Since some of the source nodes have to switch from NDF mode to $\mathrm{CF}$ for the optimum relaying strategy, the source nodes that have non-active decodability constraints with the optimum power allocation of $A_{k, D F}=A_{k, C F-s t r i c t}^{\prime}$ and $A_{k, D F}=A_{k, C F-\text { strict }}$ will be allocated less relay power for the optimum relaying strategy, and the decodability constraints of these source nodes will still be non-active for the optimum relaying strategy. These source nodes should be relayed with NDF relaying also for the optimum relaying strategy selection, and will be denoted as $A_{k, N D F-s t r i c t}$ in the sequel. Using this observation, we obtain the optimum relaying strategies for the source nodes in $A_{k, C F-s t r i c t}$ and $A_{k, D F-\text { strict }}$. Furthermore, we propose the following strategy to enable switching from NDF to $\mathrm{CF}$ for the source nodes that are neither in $A_{k, C F-s t r i c t}$ nor $A_{k, N D F-s t r i c t}$. Noting that the cost of switching from NDF to CF of a source node in terms of power consumption is $P_{r i-t h r e 2}-P_{r i-t h r e 1}$, we choose the source node that has the lowest $P_{r i-t h r e 2}-P_{r i-t h r e 1}$ for switching from NDF to $\mathrm{CF}$, and check if the optimum power allocation for such a switch in the relaying strategy results in increased sum capacity. We continue to switch the source nodes to $\mathrm{CF}$ until switching a source node from NDF to $\mathrm{CF}$ does not improve the sum capacity or all source nodes are switched to $\mathrm{CF}$ except the source nodes in $A_{k, N D F-s t r i c t}$. The outline of the near-optimum relaying strategy selection algorithm (NORSS) is summarized in Table I.

\section{NumericAl RESUlts}

In this section, we present numerical results to demonstrate the performance of optimum power allocation and relaying strategy selection for a relay assisted F/TDMA ad hoc network. For numerical results, we consider an ad hoc F/TDMA multiuser relay network with 4 source nodes and

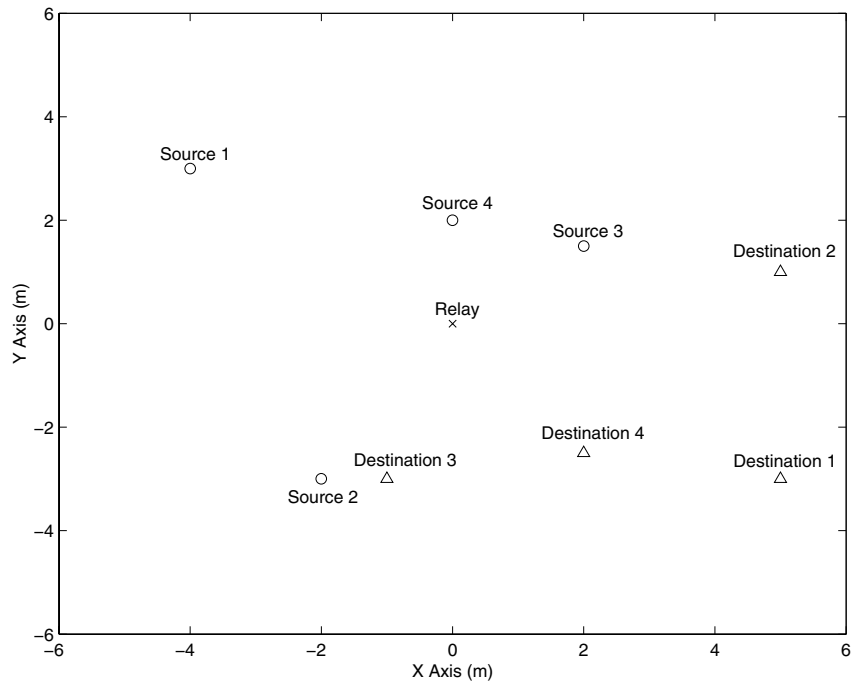

Fig. 3. 4 User Relay Assisted Ad hoc Network

one relay node that serves all (Figure 3 ) in one of the RDF, NDF, AF, CF relaying strategies for each source node. The link SNRs of the source nodes used throughout the simulations are inversely proportional to the cube of the distance between the nodes, and are $\left\{\left(P_{s i} \beta_{i}^{2}, P_{s i} \alpha_{i}^{2}, \gamma_{i}^{2}\right)\right\}_{i=1}^{4}=$ $\{(-1.02,9.03,7.03),(2.81,13.29,8.78),(8.01,18.06,15.00)$, $(9.23,20.96,14.84)\} \mathrm{dB}$. We investigate the individual capacities and the sum capacities resulting from the proposed by hybrid relaying strategy selection algorithm (NORSS) for a range of relay power constraints. Figure 4 shows the performance of the individual capacities of the source nodes achieved by NORSS with optimum power allocation. We observe that, in the low relay power case, source nodes 3 and 4 are assisted in NDF relaying strategy whereas no relay power is dedicated to the 1 st and 2 nd source nodes. This is due to the fact that the source nodes 3 and 4 have much better relay to destination links than the source nodes 1 and 2 . Thus, it is not efficient to allocate power to the source nodes 1 and 2 in low relay power case. As the available power at the relay node increases, the relay node starts to help the 1st and 2nd source nodes. We observe that after some threshold, the potentials of the source nodes for NDF relaying strategy have been reached, and the relay node switches to $\mathrm{CF}$ relaying strategy after enough relay power becomes available for each source node. As expected in low relay power cases, NDF relaying strategy is preferred whereas in high relay power cases, the relay node switches to $\mathrm{CF}$ relaying strategy to provide higher capacities. Table II shows the limits of the individual capacities of the source nodes obtained by direct transmission, RDF/NDF assisted transmission (7) and AF/CF assisted transmission (13). We observe that the individual capacities of the source nodes can be improved by using relay assistance. Figure 5 demonstrates the sum capacities resulting from NORSS, pure RDF, NDF, AF and CF relaying strategies with optimum power allocation. As expected NORSS uses the advantages of both NDF and CF relaying, and performs better than pure RDF, NDF, AF or CF relaying. Note that for low relay power cases, hybrid relaying favors NDF, and 


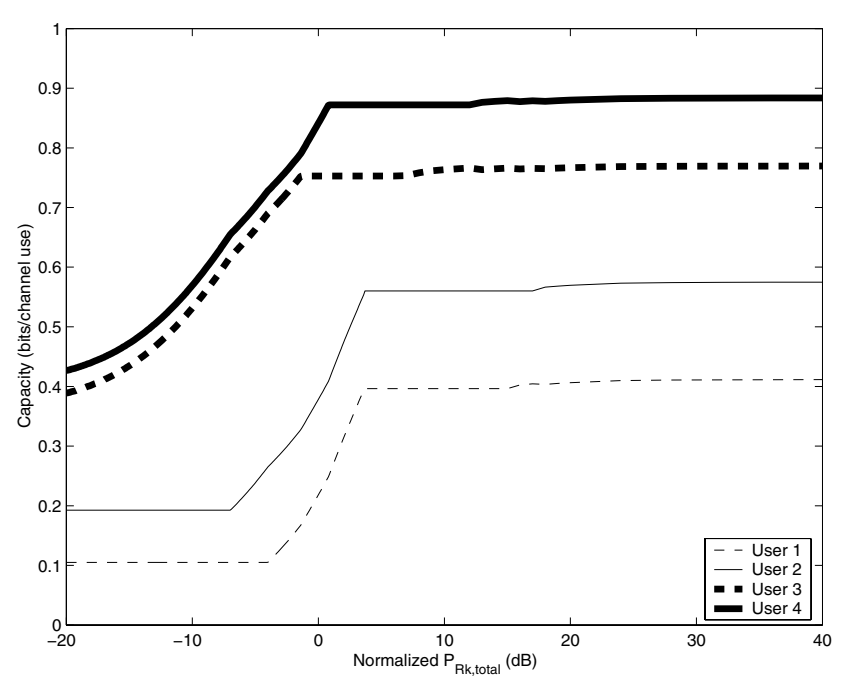

Fig. 4. Performance of NORSS Algorithm

TABLE II

COMPARISON OF CAPACITY LIMITS IN BITS/CHANNEL USE

\begin{tabular}{|l|c|c|c|c|}
\hline \hline Transmission Scheme & User 1 & User 2 & User 3 & User 4 \\
\hline \hline Direct & 0.1201 & 0.2416 & 0.5142 & 0.5937 \\
\hline RDF/NDF & 0.3962 & 0.5602 & 0.7528 & 0.8722 \\
\hline $\mathrm{AF} / \mathrm{CF}$ & 0.4114 & 0.5749 & 0.7695 & 0.8838 \\
\hline
\end{tabular}

for high relay power cases, hybrid relaying uses $\mathrm{CF}$ for all source nodes to obtain higher sum capacities. Observe also that NORSS finds the optimum relaying strategy for each source node for the relay assisted ad hoc network considered in Figure 3.

\section{CONCLUSION}

In this paper, we have considered a two-hop multiple sourcedestination F/TDMA wireless network where intermediate nodes relay the information of source nodes. We have solved the problem of optimally allocating the power of each relay node between the source nodes' transmissions it is assisting for hybrid relay transmission schemes. We have first investigated the power allocation problem for the hybrid relay nodes when the relaying strategy of each source node is given. Motivated by higher sum capacities, we have then investigated the power allocation problem with relaying strategy selection where the hybrid relay nodes chooses the best relaying strategy for each source node, and proposed a near-optimum relaying strategy selection algorithm. We have observed that hybrid relaying with the near-optimum relaying strategy selection algorithm and optimum power allocation performs better than pure RDF, $\mathrm{NDF}, \mathrm{AF}$ or $\mathrm{CF}$ relaying with optimum power allocation.

\section{REFERENCES}

[1] S. Toumpis and A. J. Goldsmith. Capacity regions for wireless ad hoc networks. IEEE Tran. on Wireless Comm., 2(4):736 - 748, July 2003.

[2] O. Leveque and I. E. Telatar. Info-theoretic upper bounds on the capacity of large extended ad hoc wireless networks. IEEE Tran. on Info. Theory, 51(3):858 - 865, March 2005.

[3] E. van der Meulen. A survey of multi-way channels in information theory: 1961-1976. IEEE Tran. on Info. Theory, 23(1):1 - 37, Jan. 1977.

[4] T. M. Cover and A. A. El Gamal. Capacity theorems for the relay channel. IEEE Tran. on Info. Theory, 25(5):572 - 584, Sept. 1979.

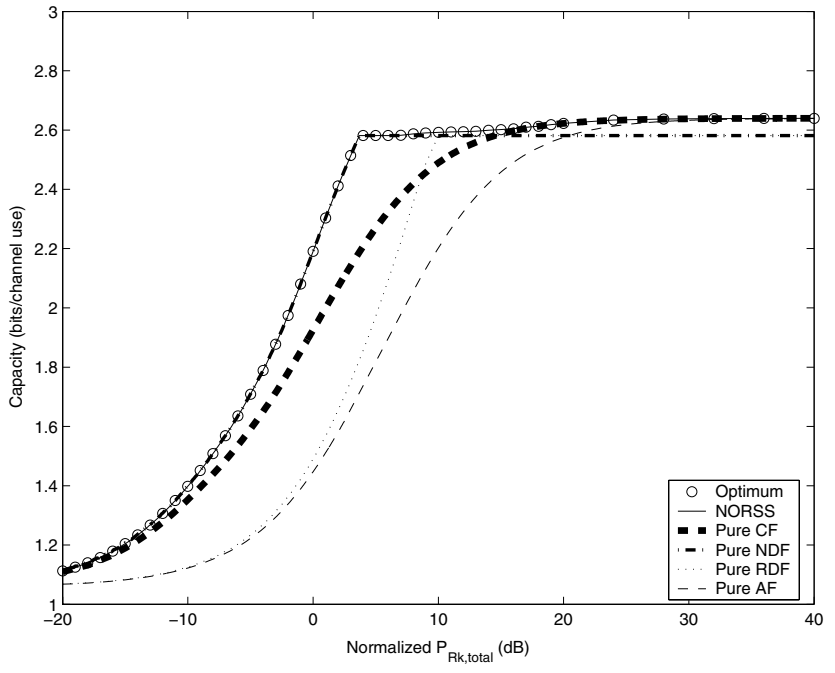

Fig. 5. Comparison of Relaying Strategies

[5] J. N. Laneman, D. N. C. Tse, and G. W. Wornell. Cooperative diversity in wireless networks: Efficient protocols and outage behavior. IEEE Tran. on Info. Theory, 50(12):3062 - 3080, Dec. 2004.

[6] A. Sendonaris, E. Erkip, and B. Aazhang. User cooperation diversity part I-II: System description and implementation, aspects and performance analysis. IEEE Tran. on Comm., 51(11):1927 - 1938, Nov. 2003.

[7] I. Maric and R. Yates. Forwarding strategies for parallel-relay networks. In Conf. on Info. Sciences and Systems, CISS'04, March 2004.

[8] I. Maric and R. D. Yates. Bandwidth and power allocation for cooperative strategies in Gaussian relay networks. In 38th Asilomar Conf. on Signals, Systems and Computers, Nov. 2004.

[9] D. R. Brown III. Resource allocation for cooperative transmission in wireless networks. In 38th Asilomar Conf. on Signals, Systems and Computers, Nov. 2004.

[10] M. O. Hasna and M. S. Alouini. Optimal power allocation for relayed transmissions over Rayleigh-fading channels. IEEE Tran. on Wireless Comm., 3(6):1999 - 2004, Nov. 2004.

[11] A. Host-Madsen and J. Zhang. Capacity bounds and power allocation in wireless relay channel. IEEE Tran. on Info. Theory, 51(6):2020 2040, June 2005.

[12] R. Pabst et al. Relay-based deployment concepts for wireless and mobile broadband radio. IEEE Comm. Magazine, 42(9):80 - 89, Sept. 2004.

[13] I. Maric and R. D. Yates. Cooperative multicast for maximum network lifetime. IEEE Journal on Selected Areas in Comm., 23(1):127 - 135, Jan. 2005.

[14] A. Scaglione and Hong Yao-Win. Opportunistic large arrays: cooperative transmission in wireless multihop ad hoc networks to reach far distances. IEEE Tran. on Signal Processing, 51(8):2082 - 2092, August 2003.

[15] P. Gupta and P. R. Kumar. Towards an information theory of large networks: an achievable rate region. IEEE Tran. on Info. Theory, 49(8):1877 - 1894, August 2003.

[16] G. Kramer, M. Gastpar, and P. Gupta. Cooperative strategies and capacity theorems for relay networks. IEEE Tran. on Info. Theory, 51(9):3037 - 3063, Sept. 2005.

[17] S. Serbetli and A. Yener. Optimum power allocation for relay assisted F/TDMA ad hoc networks. In IEEE Int. Conf. on Wireless Networks, Comm., and Mobile Computing 2005, June 2005.

[18] L. Sankaranarayanan, G. Kramer, and N. B. Mandayam. Capacity theorems for the multiple-access relay channel. In 42nd Annual Allerton Conf. on Comm., Control and Computers, Sept. 2004.

[19] A. Wyner and J. Ziv. The rate-distortion function for source coding with side information at the decoder. IEEE Tran. on Info. Theory, 22(1):1 10, Jan. 1976. 